\title{
Dritter Sitzungstag
}

Freitag, den 3. April 1959, im Deutschen Museum, Vormittagssitzung von $9.00-13.08 \mathrm{Uhr}$

Die Sitzung wird um 9.00 Uhr durch den Vorsitzenden, Prof. Dr. W. BLock, eröffnet.

Vorsitzender: Meine Damen und Herren! Wir beginnen den dritten Sitzungstag. Wir sprechen zunächst über

\section{Thoraxchirurgie}

und ich bitte hier Herrn ZenKer und Herrn Heberer.

\section{Herzoperationen mit Hilfe einer Herz-Lungen-Maschine}

Von

\author{
R. ZENkER-München und G. HeBeBer-Marburg/Lahn \\ (Vortragender: R. ZENKER) \\ Mit 10 Textabbildungen
}

Allen Teilnehmern der letztjährigen Tagung sind die Referate vor allem von Kirkin, Senning und DerRa über die Möglichkeiten der Korrektur von Herzfehlern unter Sicht mit Hilfe einer Herz-LungenMaschine oder in Hypothermie in Erinnerung. Der Durchführung von Herzoperationen in Unterkühlung auf $29-30^{\circ}$ wird zweifellos durch die Zeitnot eine Grenze gesetzt. Versuche, die reine Operationszeit bei offenem Herzen durch zusätzliche Perfusion der Koronarien oder durch Senken der Körpertemperatur auf $20-16^{0}$ von $8 \mathrm{~min}$ auf $20-60 \mathrm{~min}$ zu verlängern, haben entweder bei Eingriffen am Menschen nicht befriedigt oder sind über das Stadium des Experiments nicht hinausgediehen.

Gegenüber der Hypothermie hat der extralkorporale Kreislauf mit Hilfe einer Herz-Lungen-Maschine den großen Vorteil, auch länger dauernde Korrekturen von Herzfehlern ausführen zu können. Wie zu den beiden ersten Operationen, über die ich vor einem Jahr berichtete, haben wir auch zu den weiteren insgesamt 26 Eingriffen am Herzen die Herz-Lungen-Maschine mit dem Gibbon-Typ-Oxygenator nach KAY und Gamrtner verwendet. Diese Maschine (Abb. 1) haben meine Mitarbeiter Borst, GeHL und Schmidt-MENDE in Zusammenarbeit mit dem Physikalischen Institut der Universität Marburg/Lahn so umgebaut, daß sie absolut zuverlässig arbeitet. Auch wurde eine Vorrichtung zur Trockenfüllung angebracht, die wir bisher nur im Experiment verwendeten. Das gesamte Aggregat besteht aus der eigentlichen Herz-Lungen-Maschine, die Sie hier sehen und der Schaltung, die das nächste Diapositiv zeigen wird. 